\title{
Storage time of platelet concentrates and risk of a positive blood culture; A nationwide cohort study
}

\author{
Aukje L. Kreuger ${ }^{1,2}$; K. Rostgaard ${ }^{3}$; R.A. Middelburg ${ }^{1,2}$; J-L. H. Kerkhoffs ${ }^{1,4}$; G. Edgren ${ }^{5,6}$; C. Erikstrup ${ }^{7}$; O.B. \\ Pedersen $^{8}$; K. Titlestad ${ }^{9} ;$ K.R. Nielsen ${ }^{10}$; S.R. Ostrowski ${ }^{11}$; M.Voldstedlund ${ }^{12} ;$ J.G. van der Bom ${ }^{1,2} ; \mathrm{H}$. \\ Ullum $^{11}$; H. Hjalgrim ${ }^{3}$ \\ 1. Center for Clinical Transfusion Research, Sanquin Research, Leiden, the Netherlands \\ 2. Department of Clinical Epidemiology, Leiden University Medical Center, Leiden, the Netherlands \\ 3. Department of Epidemiology Research, Statens Serum Institut, Copenhagen, Denmark \\ 4. Haga hospital, Den Haag, the Netherlands \\ 5. Department of Medical Epidemiology and Biostatistics, Karolinska Institutet, Stockholm, Sweden \\ 6. Hematology center, Karolinska University Hospital, Stockholm, Sweden \\ 7. Department of Clinical Immunology, Aarhus University Hospital, Aarhus, Denmark \\ 8. Department of Clinical Immunology, Naestved Hospital, Naestved, Denmark \\ 9. Department of Clinical Immunology, Odense University Hospital, Odense, Denmark \\ 10. Department of Clinical Immunology, Aalborg University Hospital, Aalborg, Denmark \\ 11. Department of Clinical Immunology, the Blood Bank, Rigshospitalet, University Hospital of Copenhagen, Copenhagen, \\ Denmark \\ 12. Department of Infectious Disease Epidemiology, Statens Serum Institut, Copenhagen, Denmark
}

Running head storage time platelets and blood culture

Keywords platelet transfusion, storage time, blood culture

\section{Conflict of interest}

None of the authors report a conflict of interest relevant to the research described in this paper.

\section{Corresponding author}

Aukje Kreuger

Center for Clinical Transfusion Research

Sanquin Research, Leiden

Plesmanlaan 1a

2333 BZ Leiden

E-mail: a.l.kreuger@lumc.nl

Telephone: +31(0)715268872

Text word count: 2950

Abstract word count: 246

Figures and tables: 3 figures, 3 tables

References: 33

online appendix: 1 figure, 3 tables 


\section{Abstract}

Background Concern of transfusion-transmitted bacterial infections has been the major hurdle to extend shelf life of platelet concentrates. We aimed to investigate the association between storage time and risk of positive blood cultures at different times after transfusion.

Methods We performed a nationwide cohort study among platelet transfusion recipients in Denmark between 2010 and 2012, as recorded in the Scandinavian Donations and Transfusions (SCANDAT2) database. Linking with a nationwide database on blood cultures (MiBa), we compared the incidence of a positive blood culture among recipients of platelets stored six to seven days (old) to those receiving fresh platelets (one to five days), using Poisson regression models. We considered cumulative exposures in windows of one, three, five, and seven days.

Results A total of 9,776 patients received 66,101 platelet transfusions. The incidence rate ratio of a positive blood culture the day after transfusion of at least one old platelet concentrate was 0.77 (Cl 0.54-1.09) compared to transfusion of fresh platelet concentrates. The incidence rate of a positive blood culture was lower the day after receiving one old compared to one fresh platelet concentrate (IRR 0.57; $\mathrm{Cl}$ : 0.37-0.87). Three, five or seven days after transfusion, storage time was not associated with the risk of a positive blood culture.

Conclusion Storage of buffy coat derived platelet concentrates in PAS-C up to seven days seems safe regarding the risk of a positive blood culture. If anything, transfusion of a single old platelet concentrate may decrease this risk the following day. 


\section{Introduction}

Platelet concentrates are transfused to prevent or treat bleeding complications in patients with low platelet count or severe platelet dysfunction. In contrast to other blood components, platelet concentrates are stored at room temperature which may facilitate bacterial growth.(1) Bacterial sepsis caused by transfusion of contaminated blood products currently constitutes the largest transfusionassociated infectious risk.(2) In many blood centers, platelet concentrates are screened for bacterial contamination in an attempt to reduce this risk. $(3,4)$ However, such screening is costly and is limited by false negative test results. $(2,5)$

Because most reported septic transfusion reactions were associated with platelet concentrates stored for 4 days or more, older platelet concentrates are believed to increase the risk of transfusionassociated bacterial infections.(6-9) A reduction of the maximum permitted storage time of platelet concentrates could conceivably reduce this risk. Therefore, storage time has been limited to 3.5 days in Japan and 4 days in Germany. $(4,10)$ Such a strategy might increase rates of product outdating and limit the number of components in stock to cope with emergency situations.(11) In several countries, including the Netherlands and Denmark, platelet concentrates can be stored for up to seven days in combination with bacterial screening.(4)

Besides a direct risk of transfusion-transmitted bacterial infections, platelets could also modulate the immune response and thereby influence the risk of infections. During storage several cytokines and chemokines, which could have immunomodulatory effects, are released.(12)

We have previously shown that the overall risk of bacteremia of any cause was decreased in hematological patients one day after transfusion of platelet concentrates stored five to seven days as compared to patients who received units stored one or two days. The association was limited to transfusion of platelet concentrates stored in plasma, whereas storage time was not associated with the risk of all-cause bacteremia when the platelets were stored in platelet additive solution (PAS). However, the power of the latter analysis was limited by the sample size for platelets stored in PAS-C and the maximal storage time of five days for platelets in PAS-B.(13)

In Denmark, all platelet concentrates are stored in PAS-C with a maximal storage time of seven days. The current study aimed at investigating the effect of storage for six or seven days on risk of a positive blood culture at different times after transfusion in all recipients of a platelet transfusion by using administrative health care data. 


\section{Methods}

\section{Setting}

We performed a nationwide cohort study among all patients receiving platelet transfusions in Denmark between 2010 and 2012. For the purpose of the present study, we restricted the study population to patients who were 18 years or older at transfusion. To ensure a homogeneous patient population, hospitals administering less than 1000 platelet transfusions during the study period were excluded. The study was approved by the Danish Data Protection Agency (2015-57-0012).

\section{Data sources}

We obtained information on transfusion recipients and blood components from the Scandinavian Donations and Transfusions database (SCANDAT2), which has been described in detail elsewhere.(14) In brief, data on donations and transfusions were collected from blood banks covering all of Sweden and Denmark. Data were linked to national registers of migration, death, and hospital care, using the unique personal identification number assigned to all residents of Sweden and Denmark.

Recipient data included information on sex, blood group, dates of birth, death and migration, discharge diagnoses, and procedure codes. Data on blood components included date of donation and transfusion, type of blood component, and blood group of donor(s). For the current study we selected the transfusions to Danish residents.

Information on blood culture results was obtained from MiBa, the Danish microbiology database.(15) MiBa contains copies of reports from all Danish departments of microbiology with a sampling date between 1 January 2010 and 31 December 2012. Blood cultures were taken on clinical indication or routinely as part of certain treatment protocols, not necessarily directly related to the transfusion. This information was linked to the transfusion data via the personal identification number.

\section{Exposure}

Platelet concentrates were produced from buffy coats of four $A B O$ and Rhesus $D$ matched donors and re-suspended in platelet additive solution (PAS-C, Intersol, Fenwal ${ }^{\mathrm{TM}}$ ). Buffy coats are pooled 3 to 30 hours after donation, but preferably after overnight hold of whole blood. All platelet concentrates were screened for bacterial contamination using the BacT/Alert system, consisting of an aerobic bottle, which is inoculated with 5-10 ml. Sampling is performed right after pooling of the buffy coats. (4) Products are released according to a 'negative-to-date' procedure. Maximum storage time of platelet concentrates was seven days. Storage time was counted in days from the day of donation (day 0 ) up to and including the day of transfusion. Platelet concentrates stored for six or seven days were considered 'old' and 
platelet concentrates stored for one to five days were considered 'fresh'. Approximately $3 \%$ of all platelet concentrates were collected via apheresis. These products were taken into account when adjusting for total number of transfusions, but we did not study storage time of apheresis products, as most of these were given for specific indications.

\section{Outcome}

The primary outcome of interest was a positive blood culture, regardless of the cause. Patients could develop a positive blood culture multiple times during the study period. Two consecutive positive cultures were considered to be unrelated if separated by at least 14 days.

\section{Statistical analysis}

The main analysis tested whether the occurrence of a positive culture on a given day was associated with transfusion of at least one old platelet unit during the preceding one to seven days. We employed a sliding window approach, with an exposure ascertainment period of one, three, five, or seven days with a subsequent 1-day follow-up period during which we ascertained the occurrence of a positive blood culture(Figure 1). Patients were considered at risk if they received at least one platelet concentrate during the window period and did not have a positive blood culture within the previous 14 days. For each day of follow-up we then advanced both the exposure and outcome ascertainment periods one day at a time. Because both transfusions and blood cultures were only recorded per calendar day, it was not possible to know whether blood cultures were drawn before or after a transfusion. We therefore excluded follow-up on days on which a transfusion was given.

The incidence rate of a positive blood culture after transfusion of at least one old platelet concentrate was compared with the incidence rate after transfusion of only fresh platelet concentrates, using Poisson regression. The analyses were adjusted for day of the week, rhesus $D$ antigen positivity of the product, and hospital, using stratification of person time. Day of the week and rhesus D antigen positivity were treated as time-dependent variables, based on the last transfusion given during that window period. We did not adjust for any patient characteristic, as storage time is not known by the treating physician and therefore confounding by indication is unlikely to arise. Robust variance estimates were used, as patients could contribute more than one window period, of which each may be terminated by a positive blood culture.(16)

The number of platelet transfusions a patient received during a window period could confound the association between storage time and risk of a positive blood culture, as number of transfusions is a 
strong indicator of sickness of the patient and risk of receiving at least one old platelet concentrate. Therefore, we stratified on number of platelet transfusions: one, two, three, and four or more transfusions during the window period. For the one-day window period analyses were stratified on one, two, and three or more transfusions.

\section{Additional analyses}

We performed three additional analyses. First, we assessed the effect of receiving at least one old platelet concentrate in a subgroup of patients with a hematological malignancy or aplastic anemia. This subgroup was established using the sequential algorithm used in previous studies, based on diagnosis and procedure codes.(17-19) Here we did not differentiate between main and co-diagnoses in the hospital register data. The covariates and stratification were the same as in the main analysis.

Second, to test for a dose-response relationship, we modeled the number of old products as main exposure, stratified on number of platelet transfusions and adjusted for the same confounders as in the main analysis.

Third, we included also transfusions given during the follow-up days in the analysis to investigate whether we introduced selection bias by excluding these days. Specifically, it has been suggested that the time to the next transfusion is shorter after transfusion of an old platelet concentrate than after transfusion of a fresh concentrate.(20-22) Therefore, we could have excluded more follow-up time, and thereby probably more events, after transfusion of old platelet concentrates, which could have introduced selection bias.

All statistical analyses were conducted using SAS, version 9.4 (SAS Institute Inc., Cary, North Carolina), using the GENMOD procedure. For stratification and aggregation of follow-up time the stratify macro was used.(23) 


\section{Results}

\section{Patient characteristics}

Between 2010 and 2012, a total of 12,529 patients received at least one platelet transfusion in Denmark. Of these, 826 patients were excluded based on age at time of transfusion and 1,927 patients were excluded as they only received a transfusion in a hospital that accounted for fewer than 1000 platelet transfusions in the study period. The final cohort consisted of 9,776 patients, more men than women (62.3\% versus 37.7\%), with an average age of 64.1 years (Table 1 ). During the study period, these patients received 66,101 platelet concentrate transfusions, of which 22,240 units $(33.6 \%)$ were stored for six or seven days. This relatively large proportion of old platelet concentrates is a consequence of the first-in-first-out policy. Forty-nine percent of all platelet concentrates were transfused to patients with a hematological malignancy, $15.6 \%$ to patients with trauma or burns, and $8.6 \%$ to patients who underwent cardiothoracic surgery. The distribution of diagnoses was similar among the storage time categories. Information about blood group of the product was missing for 11,156 products, but this was equally distributed among the storage time categories. The proportion of rhesus $D$ negative products increased with increasing storage time (Table 2).

\section{$\underline{\text { Incidence of positive blood cultures }}$}

The day after transfusion 211 cases of positive blood cultures occurred, which corresponds to an incidence rate of 8.0 per 1000 observation days (95\% confidence interval (Cl) 7.0 to 9.2). Among patients receiving a transfusion within the last three days, the incidence rate was 6.5 per 1000 days $(95 \% \mathrm{Cl} 5.9$ to 7.2). Considering a five day window period the incidence rate was 5.7 per 1000 days $(95 \% \mathrm{Cl} 5.2$ to 6.2) and for the seven day window period this was 5.2 per 1000 days ( $95 \% \mathrm{Cl} 4.8$ to 5.7). The incidence rate increased with an increasing number of platelet transfusions in all window periods (Figure 2).

\section{Old versus fresh platelet concentrates}

Figure 3 presents the incidence rate ratios (IRR) of a positive blood culture after transfusion of at least one old platelet concentrate, compared to only fresh platelet concentrates, for all window periods, stratified on total number of platelet transfusions. The incidence rate ratio of a positive blood culture the day after transfusion of at least one old platelet concentrate compared to only fresh platelet concentrates was 0.77 (95\% $\mathrm{Cl} 0.54$ to 1.09). Considering a window period of three days, the incidence rate ratio of a positive blood culture was 0.96 ( $95 \% \mathrm{Cl} 0.76$ to 1.23$)$. This was $0.98(95 \% \mathrm{Cl} 0.79$ to 1.21$)$ for a five day window period and 1.05 ( $95 \% \mathrm{Cl} 0.87$ to 1.28$)$ for a seven day window period (supplemental data). 
For patients receiving a single platelet concentrate, the incidence rate of a positive blood culture the day after transfusion was lower if this was an old platelet concentrate (IRR $0.57 ; 95 \% \mathrm{Cl} 0.37-0.87$ ). This association was not statistically significant if the old platelet concentrate was transfused in the preceding three, five or seven days (Figure 3).

\section{Additional analyses}

For patients with a hematological malignancy or aplastic anemia, the incidence rate ratio of bacteremia the day after transfusion of at least one old platelet concentrate was 0.54 ( $\mathrm{Cl} 0.31$ to 0.87 ) compared to transfusion of only fresh platelet concentrates. After receiving a single old, compared to a single fresh, platelet concentrate the incidence rate ratio was $0.44(95 \% \mathrm{Cl} 0.26$ to 0.76$)$. If a patient received one old platelet concentrate in the preceding three, five, or seven days, the estimates were similar, although not statistically significant (Figure 3, panel B and supplemental data).

There was no evidence of a dose-response relationship (Table 3). For patients who received two transfusions, the incidence rate ratio for a positive blood culture the day after receiving exclusively old platelet concentrates compared to exclusively fresh platelet concentrates was 1.11 ( $95 \% \mathrm{Cl} 0.53$ to 2.31). Similar estimates were observed for patients receiving three, or four or more platelet concentrates and if these transfusions were given during a longer window period (Table 3).

Including the follow-up days on which a patient had received a platelet transfusion did not change the results (supplemental data). 


\section{Discussion}

In this nationwide cohort study, transfusion with platelet concentrates stored for six or seven days was not associated with an increased risk of a positive blood culture compared to transfusion with platelet concentrates stored for five days or less. If anything, risk of a positive blood culture was lower the day after transfusion of one old platelet concentrate compared to one fresh platelet concentrate.

Storage time was only associated with a lower incidence of a positive blood culture after transfusion of a single platelet concentrate. The lack of an association when patients had received multiple transfusions might conceivably be attributable to effect modification by indication or underlying morbidity.

Specifically, diagnoses and indications might differ between patients who need only one transfusion and those who need more transfusions. As corollary, the patients who needed the most transfusions might also have a higher baseline risk of infection. The increased incidence of a positive blood culture with an increasing number of transfusions, as observed in the present study, would be compatible with this notion. Hence, under such circumstances any variation in infection risk by storage time of a transfused product might be clinically irrelevant and immeasurable. In addition, the groups receiving multiple transfusions were smaller which limits the power to detect such small effects.

The protective effect of an old platelet concentrate was only seen after the shortest window period. Most hematological patients receive prophylactic antibiotics, which could result in negative blood cultures after three or more days, but maybe not immediately the day after transfusion. The higher risk of a positive blood culture soon after transfusion of fresh platelets could be due to contamination which was not yet detected by the bacterial screening system. Not all bacteria are able to proliferate within a platelet concentrate, so during storage a blood component could auto-sterilize.(2, 24, 25) However, studies have shown that platelet concentrates transfused before the screening turned out positive only marginally increase the risk of clinically significant infections. $(26,27)$ Another postulated theory is that platelets play a role in the immune system and transfusions could modulate this response. $(12,28,29)$ During storage, efficacy of platelets reduces: referred to as 'the storage lesion'.(30, 31) This may imply that older platelets are not consumed immediately in hemostatic activities and still exert a relatively higher activity of non-hemostatic functions that may protect the patient better against infections.

The reduction in efficacy during storage could also have introduced selection bias, as we excluded follow-up time in which a patient received a transfusion and the interval between transfusions may be shortened after transfusion of old platelet concentrates.(32) However, the sensitivity analysis including follow-up time in which a patient received a transfusion yielded similar results. 
A major strength of our study is that we were able to study storage of platelet concentrates for up to seven days. In many countries storage is limited to five days, but blood banks worldwide are seeking to extend their maximum storage time.(33) The draft guidance of the FDA stated that transfusion of platelet concentrates stored for six or seven days is allowed, provided that these concentrates are cultured again on day four or five of storage, or rapid testing is performed within 24 hours prior to transfusion. However, no culture system has been certified up to now.(34)

Studies regarding transfusion-associated sepsis are often based on data gathered by passive surveillance. It has been suggested that such a strategy underestimates the true incidence as much as 40 -fold. $(35,36)$ We included all positive blood cultures as a surrogate outcome to overcome this underestimation. Such a strategy implicates that we also included blood cultures that were positive due to contamination of the culture and not the result of a bacteremia accompanied by clinically relevant symptoms. Bacteria identified in contaminated blood cultures are often skin derived and the same as those identified in contaminated blood products and transfusion-transmitted infections.(27) It is therefore impossible to distinguish between these. As a consequence, the incidence in our study overestimates the true incidences of all-cause bacteremia and transfusion transmitted bacterial infections. As contamination of blood cultures is unrelated to storage time of platelet concentrates, this misclassification may have biased the results towards the null, meaning no association. Moreover, since we used positive blood cultures as a surrogate outcome for bacteremia, we are unable to completely rule out the potentially fatal residual risk of septic transfusion reactions after screening. This especially accounts for older products. Sampling for the BacT/Alert is performed 24 hours after donation, so initial low inocula could be missed, resulting in false negative screening. Proliferation during storage may result in a high bacterial load at time of transfusion and an increased risk of severe septic transfusion reactions. $(37,38)$ The same kind of bias applies to transfusion of other blood products. It has been suggested that red cell transfusions also have immunomodulatory effects. However, transfusion of other blood products is not associated with storage time of platelet concentrates. $(32,39)$ Therefore, these additional transfusions could not confound our results.

These biases could potentially explain the lack of an association in patients who received multiple platelet concentrates. However, they are unlikely explanations of our observation of a protective effect of transfusion of a single old platelet concentrate on the incidence of positive blood cultures.

The present findings are consistent with the results of our previous study. In a population of Dutch hematological patients, the risk of bacteremia was lower the day after transfusion of platelet 
concentrates stored in plasma for five to seven days compared to those stored one or two days.(13) In the Dutch study, we only included days on which a patient received platelet concentrates exclusively of a single storage time category. On most analyzed days, patients received only one transfusion.

Therefore, these results are comparable with the conclusion of the current study regarding transfusion of a single old platelet concentrate. Since we now have found a similar effect in two independent cohorts, using different methods, it is unlikely that this association has arisen from chance alone.

To conclude, regarding the risk of a positive blood culture, it seems to be safe to store platelet concentrates up to seven days in combination with $100 \%$ screening. Transfusion of a single old platelet concentrate may decrease the risk of a positive blood culture the day after transfusion, especially in patients with a hematological malignancy. 


\section{Authorship contributions}

A.L. Kreuger

K. Rostgaard

R.A. Middelburg

J-L. H. Kerkhoffs

G. Edgren

C. Erikstrup

O.B. Pedersen

K.Titlestad

K.R. Nielsen

S.R. Ostrowski

M. Voldstedlund

J.G. van der Bom

H. Ullum

H. Hjalgrim
Designed research, performed research, analyzed and interpreted data, and wrote the manuscript

Designed research, analyzed and interpreted data, and revised the manuscript

Designed research, interpreted data, and revised the manuscript

Designed research, interpreted data, and revised the manuscript

Collected data, and revised the manuscript

Collected data, and revised the manuscript

Collected data, and revised the manuscript

Collected data, and revised the manuscript

Collected data, and revised the manuscript

Interpreted data, and revised the manuscript

Collected data, and revised the manuscript

Designed research, interpreted data, and revised the manuscript

Designed research, interpreted data, and revised the manuscript

Designed research, interpreted data, and revised the manuscript

\section{Transparancy}

Aukje Kreuger affirms that the manuscript is an honest, accurate, and transparent account of the study being reported; no important aspects of the study have been omitted.

\section{Role of funding source}

This work was supported by funds from the Leiden University Fund /Van Trigt, the Foundation "De Drie Lichten" and the Foundation "Het Scholten-Cordes fonds" in The Netherlands. The funding sources had no role in the design and conduct of the study; collection, management, analysis, and interpretation of the data; preparation, review, or approval of the manuscript; or the decision to submit the manuscript for publication. 


\section{References}

1. Kaufman RM, Djulbegovic B, Gernsheimer T, et al. Platelet transfusion: a clinical practice guideline from the AABB. Ann Intern Med. 2015;162(3):205-13. doi: 10.7326/M14-1589.

2. Palavecino EL, Yomtovian RA, Jacobs MR. Bacterial contamination of platelets. Transfus Apher Sci. 2010;42(1):71-82.

3. Tomasulo P, Su L. Is it time for new initiatives in the blood center and/or the hospital to reduce bacterial risk of platelets? Transfusion. 2011;51(12):2527-33.

4. Pietersz RN, Reesink HW, Panzer S, et al. Bacterial contamination in platelet concentrates. Vox Sang. 2014;106(3):256-83.

5. Brecher ME, Holland PV, Pineda AA, et al. Growth of bacteria in inoculated platelets: implications for bacteria detection and the extension of platelet storage. Transfusion. 2000;40(11):1308-12.

6. Eder AF, Kennedy JM, Dy BA, et al. Bacterial screening of apheresis platelets and the residual risk of septic transfusion reactions: the American Red Cross experience (2004-2006). Transfusion. 2007;47(7):1134-42.

7. Jacobs MR, Smith D, Heaton WA, et al. Detection of bacterial contamination in prestorage culture-negative apheresis platelets on day of issue with the Pan Genera Detection test. Transfusion. 2011;51(12):2573-82.

8. Dumont L, Kleinman S, Murphy JR, et al. Screening of single-donor apheresis platelets for bacterial contamination: the PASSPORT study results. Transfusion. 2010;50(3):589-99.

9. McDonald C, Allen J, Brailsford S, et al. Bacterial screening of platelet components by National Health Service Blood and Transplant, an effective risk reduction measure. Transfusion. 2017;57(5):1122-31.

10. Vollmer T, Engemann J, Kleesiek K, et al. Bacterial screening by flow cytometry offers potential for extension of platelet storage: results of 14 months of active surveillance. Transfus.Med. 2011;21(3):175-82.

11. Veihola $\mathrm{M}$, Aroviita $\mathrm{P}$, Linna $\mathrm{M}$, et al. Variation of platelet production and discard rates in 17 blood centers representing 10 European countries from 2000 to 2002. Transfusion. 2006;46(6):991-5.

12. Cognasse $F$, Boussoulade $F$, Chavarin $P$, et al. Release of potential immunomodulatory factors during platelet storage. Transfusion. 2006;46(7):1184-9.

13. Kreuger AL, Middelburg RA, Bank CMC, et al. Storage time of platelet concentrates and all-cause bacteremia in hematological patients. Transfusion.

14. Edgren G, Rostgaard K, Vasan SK, et al. The new Scandinavian Donations and Transfusions database (SCANDAT2): a blood safety resource with added versatility. Transfusion. 2015;55(7):1600-6.

15. Voldstedlund M, Haarh M, Molbak K. The Danish Microbiology Database (MiBa) 2010 to 2013. Euro Surveill. 2014;19(1).(pii):20667.

16. Zou G. A modified poisson regression approach to prospective studies with binary data. Am J Epidemiol. 2004;159(7):702-6.

17. Hjalgrim H, Edgren $G$, Rostgaard K, et al. Cancer incidence in blood transfusion recipients. J Natl Cancer Inst. 2007;99(24):1864-74.

18. Edgren $\mathrm{G}$, Hjalgrim $\mathrm{H}$, Reilly $\mathrm{M}$, et al. Risk of cancer after blood transfusion from donors with subclinical cancer: a retrospective cohort study. Lancet. 2007;369(9574):1724-30.

19. Shanwell A, Andersson TM, Rostgaard K, et al. Post-transfusion mortality among recipients of ABO-compatible but non-identical plasma. Vox Sang. 2009;96(4):316-23. 
20. Heuft HG, Goudeva L, Krauter J, et al. Effects of platelet concentrate storage time reduction in patients after blood stem cell transplantation. Vox Sang. 2013;105(1):18-27.

21. Slichter SJ, Davis K, Enright $\mathrm{H}$, et al. Factors affecting posttransfusion platelet increments, platelet refractoriness, and platelet transfusion intervals in thrombocytopenic patients. Blood. 2005;105(10):4106-14.

22. Akkok CA, Brinch L, Lauritzsen GF, et al. Clinical effect of buffy-coat vs. apheresis platelet concentrates in patients with severe thrombocytopenia after intensive chemotherapy. Vox Sang. 2007;93(1):42-8.

23. Rostgaard K. Methods for stratification of person-time and events - a prerequisite for Poisson regression and SIR estimation. Epidemiol Perspect Innov. 2008;5:7.(doi):10.1186/742-5573-5-7.

24. Muller TH, Montag T, Seltsam AW. Laboratory Evaluation of the Effectiveness of Pathogen Reduction Procedures for Bacteria. Transfus Med Hemother. 2011;38(4):242-50.

25. Stormer M, Kleesiek K, Dreier J. Propionibacterium acnes lacks the capability to proliferate in platelet concentrates. Vox Sang. 2008;94(3):193-201.

26. Koopman MM, van't Ende E, Lieshout-Krikke R, et al. Bacterial screening of platelet concentrates: results of 2 years active surveillance of transfused positive cultured units released as negative to date. Vox Sang. 2009;97(4):355-7.

27. Walther-Wenke G, Schrezenmeier H, Deitenbeck R, et al. Screening of platelet concentrates for bacterial contamination: spectrum of bacteria detected, proportion of transfused units, and clinical follow-up. Ann Hematol. 2010;89(1):83-91.

28. Semple JW, Italiano JE, Jr., Freedman J. Platelets and the immune continuum. Nat Rev Immunol. 2011;11(4):264-74.

29. Geiger TL. Transfusion-associated immune modulation: a reason to TRIM platelet transfusions? Transfusion. 2008;48(9):1772-3.

30. Sahler J, Grimshaw K, Spinelli SL, et al. Platelet storage and transfusions: new concerns associated with an old therapy. Drug Discov Today Dis Mech. 2011;8(1-2):e9-e14.

31. Caram-Deelder C, Kreuger AL, Jacobse J, et al. Effect of platelet storage time on platelet measurements: a systematic review and meta-analyses. Vox Sang. 2016;111(4):374-82.

32. Kreuger AL, Caram-Deelder C, Jacobse J, et al. Effect of storage time of platelet products on clinical outcomes after transfusion: a systematic review and meta-analyses. Vox Sang 2017;112(4):291-300.

33. Dunbar NM, Dumont LJ, Szczepiorkowski ZM. How do we implement Day 6 and Day 7 platelets at a hospital-based transfusion service? Transfusion. 2016;56(6):1262-6.

34. Bacterial Detection Testing by Blood Collection Establishments and Transfusion Services to Enhance the Safety and Availability of Platelets for Transfusion. Accessed at US Food and Drug Administration at http://www.fda.gov/downloads/BiologicsBloodVaccines/GuidanceComplianceRegulatorylnform ation/Guidances/Blood/UCM425952.pdf on July 2016.

35. Hong $\mathrm{H}$, Xiao $\mathrm{W}$, Lazarus $\mathrm{HM}$, et al. Detection of septic transfusion reactions to platelet transfusions by active and passive surveillance. Blood. 2016;127(4):496-502.

36. Rogers MA, Rohde JM, Blumberg N. Haemovigilance of reactions associated with red blood cell transfusion: comparison across 17 Countries. Vox Sang. 2016;110(3):266-77.

37. Benjamin RJ, Wagner SJ. The residual risk of sepsis: modeling the effect of concentration on bacterial detection in two-bottle culture systems and an estimation of false-negative culture rates. Transfusion. 2007;47(8):1381-9.

38. Jacobs MR, Good CE, Lazarus HM, et al. Relationship between bacterial load, species virulence, and transfusion reaction with transfusion of bacterially contaminated platelets. Clin Infect Dis. 2008;46(8):1214-20. 
39. Rohde JM, Dimcheff DE, Blumberg N, et al. Health care-associated infection after red blood cell transfusion: a systematic review and meta-analysis. Jama. 2014;311(13):1317-26. 
Table 1. Characteristics of study population

\begin{tabular}{ll}
\hline & Number of patients (\%) \\
\hline Patients, $\mathbf{n}$ & 9,776 \\
Male, $\mathbf{n}(\%)$ & $6,088(62.3)$ \\
Age, $\mathbf{n}(\%)$ & \\
$\mathbf{1 8 - 4 9}$ years & $1,533(15.7)$ \\
$\mathbf{5 0 - 7 4}$ years & $6,011(61.5)$ \\
$\mathbf{2 7 5}$ year & $2,232(22.8)$ \\
Mean (SD) age in years & $64.1(14.6)$ \\
Median (IQR) number of transfused platelet concentrates & $2(1-6)$ \\
Median (IQR) number of transfused red blood cell concentrates & $12(5-24)$ \\
Median (IQR) number of transfused plasma products & $2(0-8)$ \\
\hline
\end{tabular}


Table 2. Characteristics of transfused platelet concentrates

\begin{tabular}{llll}
\hline & Storage time 1-5 days & Storage time 6 -7 days & Total \\
\hline $\begin{array}{l}\text { Number of platelet } \\
\text { concentrates (\%)* }\end{array}$ & $34,722(52.5)$ & $22,240(33.6)$ & $66,101(100)$ \\
$\begin{array}{l}\text { Male, } \mathbf{n}(\%)+ \\
\text { Mean (SD) age in yearst }\end{array}$ & $21,628(62.3)$ & $14,151(63.6)$ & $41692(63.1)$ \\
Median (IQR) number of prior & $60.3(14.9)$ & $60.0(15.1)$ & $60.2(14.9)$ \\
transfusions all products & $32(9-79)$ & $33(9-83)$ & $34(10-84)$ \\
Main indication & & & \\
$\quad$ Hematology & $17,029(49.0)$ & $10,681(48.0)$ & $32,547(49.2)$ \\
$\quad$ Cardiothoracic surgery & $3,017(8.7)$ & $1,914(8.6)$ & $5,657(8.6)$ \\
$\quad$ Trauma and burns & $5,407(15.6)$ & $3,429(15.4)$ & $10,319(15.6)$ \\
$\quad$ Bleeding & $1,177(3.4)$ & $675(3.0)$ & $2,106(3.2)$ \\
$\quad$ Unknown & $8,092(23.3)$ & $5,541(25.0)$ & $15,472(23.4)$ \\
Donor ABO blood group, $\mathbf{n}(\%)$ & & & \\
$\quad$ A & $13,958(40.2)$ & $8,019(36.1)$ & $22,377(33.9)$ \\
$\quad$ B & $1,551(4.5)$ & $510(2.3)$ & $2,084(3.2)$ \\
$\quad$ AB & $9(0.03)$ & $1(0.0)$ & $15(0.0)$ \\
$\quad$ Rhesus antigen positivity, $\mathbf{n}(\%)$ & $26,949(77.6)$ & $12,216(54.9)$ & $30,469(46.1)$ \\
Missing blood group, $\mathbf{n}(\%)$ & $2,468(7.1)$ & $14,094(63.4)$ & $42,065(63.6)$ \\
\hline
\end{tabular}

* Percentages do not add up until 100\%. In 'total' are also apheresis products (2.7\%) and products with unknown storage time (11.1\%) included.

† Expressed per number of platelet concentrates 
Table 3. Incidence rate ratio for positive blood culture per number of old platelet concentrates, stratified on total number of platelet transfusions during a window period of one, three, five, or seven days.

Incidence rate ratios with 95\% confidence intervals are adjusted for hospital, rhesus D blood group, and day of the week. Transfusion of only fresh platelet concentrates were used as reference. Incidences are depicted in table 2 of the online appendix.

\begin{tabular}{ll|llll}
$\begin{array}{l}\text { Total } \\
\text { number of } \\
\text { platelet } \\
\text { transfusions }\end{array}$ & $\begin{array}{l}\text { Number of } \\
\text { old platelet } \\
\text { concentrates }\end{array}$ & $\mathbf{1}$ day & $\mathbf{3}$ days & $\mathbf{5}$ days & $\mathbf{7}$ days \\
\hline 1 & 0 & ref & ref & ref & ref \\
1 & 1 & $0.57(0.37-0.87)$ & $0.87(0.62-1.22)$ & $0.79(0.57-1.08)$ & $0.87(0.64-1.18)$ \\
\hline 2 & 0 & $r e f$ & $r e f$ & $r e f$ & $r e f$ \\
2 & 1 & $1.54(0.64-3.71)$ & $0.87(0.53-1.44)$ & $0.92(0.58-1.43)$ & $1.08(0.72-1.63)$ \\
2 & 2 & $1.11(0.53-2.31)$ & $0.98(0.62-1.54)$ & $0.88(0.55-1.42)$ & $0.95(0.62-1.47)$ \\
\hline $3^{*}$ & 0 & ref & $r e f$ & $r e f$ & $r e f$ \\
$3^{*}$ & 1 & $2.39(0.40-14.31)$ & $1.05(0.39-2.81)$ & $1.30(0.65-2.61)$ & $1.09(0.59-2.02)$ \\
$3^{*}$ & 2 & $5.10(1.13-23.00)$ & $1.87(0.75-4.63)$ & $1.88(0.97-3.65)$ & $1.24(0.65-2.36)$ \\
$3^{*}$ & 3 & $1.94(0.43-8.71)$ & $0.21(0.03-1.63)$ & $0.79(0.29-2.18)$ & $0.87(0.36-2.09)$ \\
\hline$\geq 4$ & 0 & N/A & ref & ref & ref \\
$\geq 4$ & 1 & N/A ${ }^{\dagger}$ & $1.10(0.35-3.41)$ & $1.18(0.62-2.23)$ & $1.07(0.59-1.91)$ \\
$\geq 4$ & 2 & N/A & $1.40(0.52-3.78)$ & $0.84(0.42-1.68)$ & $1.08(0.61-1.91)$ \\
$\geq 4$ & 3 & N/A & $0.76(0.17-3.42)$ & $1.03(0.49-2.14)$ & $1.26(0.68-2.33)$ \\
$\geq 4$ & $\geq 4$ & N/A & $1.64(0.70-3.80)$ & $1.20(0.66-2.17)$ & $1.37(0.81-2.33)$
\end{tabular}

* For the 1 day window period. the total number of platelet transfusions is 3 or more, as hardly any patient received 4 or more transfusions within 1 day.

† Not applicable, insufficient number of patients received $\geq 4$ transfusions in the 1 day window period. 
Figure 1. Examples of cumulative exposure during a seven-day window period

A) All transfusions during the window period are counted. Day 8 is the day of follow-up.

B) The next window period starts one day later.

C) If a transfusion is given at the follow-up day, this window period is excluded.

D) Positive blood culture at day of follow-up

E) After a positive blood culture patients are censored for 14 days.

A) \begin{tabular}{|l|l|l|l|l|l|l|l|l|}
\hline $\mathrm{T}$ & & $\mathrm{T}$ & & $\mathrm{T}$ & & $\mathrm{T}$ & $\mathrm{FU}$ & 4 transfusions \\
\hline
\end{tabular}

B) \begin{tabular}{|l|l|l|l|l|l|l|l|l|l|}
\hline $\mathrm{T}$ & & $\mathrm{T}$ & & $\mathrm{T}$ & & $\mathrm{T}$ & & $\mathrm{FU}$ & 3 transfusions \\
\hline
\end{tabular}

C) \begin{tabular}{|l|l|l|l|l|l|l|l|l|l|l|}
\hline $\mathrm{T}$ & & $\mathrm{T}$ & & $\mathrm{T}$ & & $\mathrm{T}$ & & & $\mathrm{T}$ excl & excluded, transfusion given during follow-up
\end{tabular}

D) \begin{tabular}{|l|l|l|l|l|l|l|l|l|l|l|l|}
\hline $\mathrm{T}$ & & $\mathrm{T}$ & & $\mathrm{T}$ & & $\mathrm{T}$ & & & $\mathrm{T}$ & $\mathrm{BC}$ & 3 3 transfusions, positive blood culture \\
\hline
\end{tabular}

E) \begin{tabular}{|l|l|l|l|l|l|l|l|l|l|l|l|l|}
\hline $\mathrm{T}$ & & $\mathrm{T}$ & & $\mathrm{T}$ & & $\mathrm{T}$ & & & $\mathrm{T}$ & $\mathrm{BC}$ & $\mathrm{X}$ & censored next 14 days \\
\hline
\end{tabular}

\begin{tabular}{|l|l|l|l|l|l|l|l|l|l|l|l|}
1 & 2 & 3 & 4 & 5 & 6 & 7 & 8 & 9 & 10 & 11 & 12 \\
\hline
\end{tabular}


Figure 2. Crude estimates of incidence rates of a positive blood culture after transfusion of 1, 2, 3 and $\geq 4$ platelet concentrates during different window periods.

The number of analyzed patients and the total follow-up time differed among the window periods, since patients were only considered at risk if they received at least one platelet concentrate during the window period and no transfusion at the day of follow-up. This means that in the window period of one day, a transfusion contributed only to one window period and one day of follow-up was counted. In a sliding window period of seven days with steps of one day, a day of transfusion contributed to seven subsequent window periods, and seven days of follow-up could be counted.

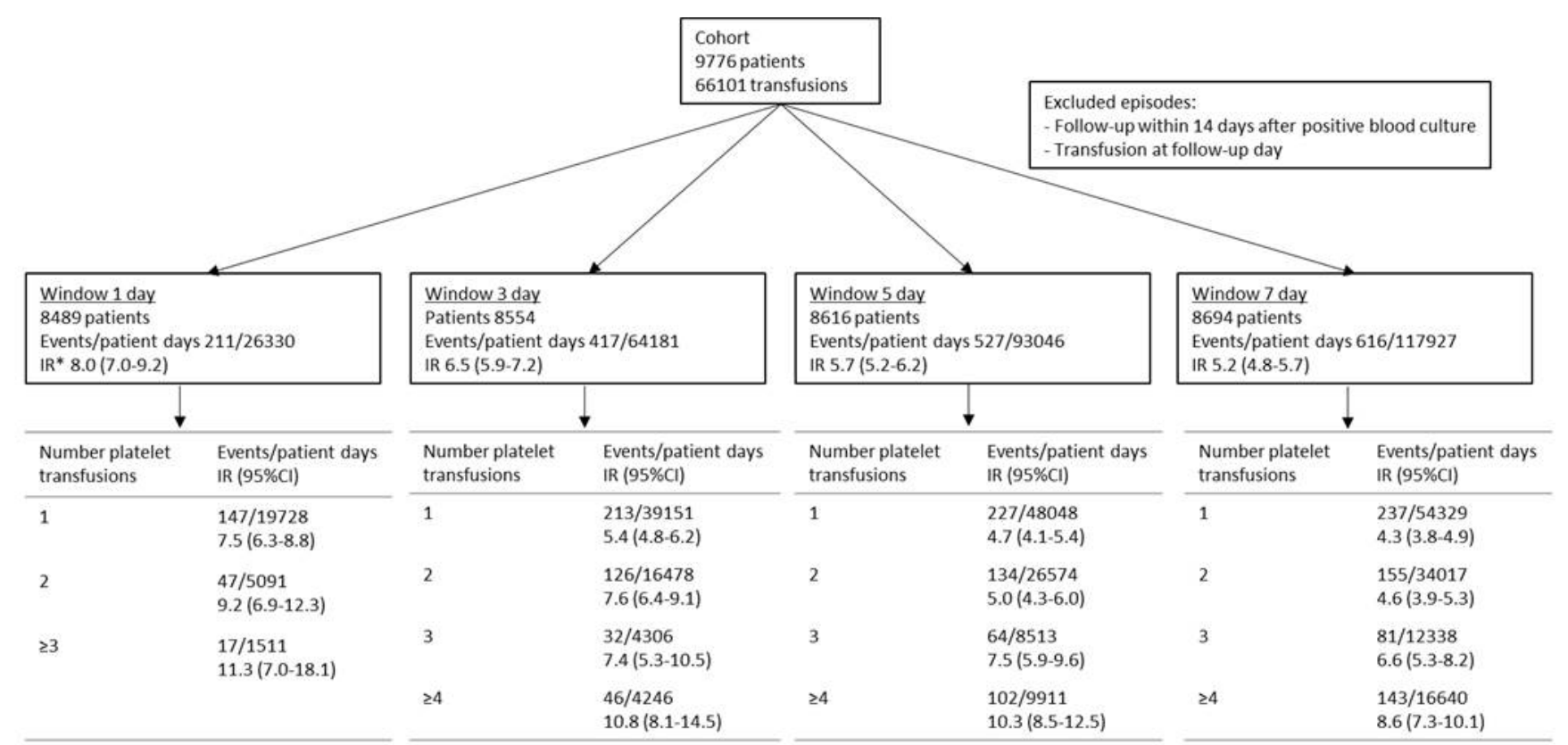

*IR (CI), incidence rate, expressed per 1000 patient-days of follow-up. 
Figure 3. Incidence rate ratio of a positive blood culture after receiving at least one old platelet concentrate compared to transfusion of only fresh platelet concentrates.

IRRs with $95 \%$ confidence interval are presented overall and stratified by number of total platelet transfusions during a window period of one, three, five, and seven days. If a patient received several platelet concentrates during the window period, no differentiation was made whether only one or more products were old. Incidence rate ratios are adjusted for hospital, rhesus D blood group, and day of the week. Overall estimate is also adjusted for number of platelet transfusions $(1,2,3, \geq 4)$.

Panel A) Entire cohort

Panel B) Patients with hematological malignancy or aplastic anemia

The corresponding numbers are given in the online appendix.

A) Entire cohort

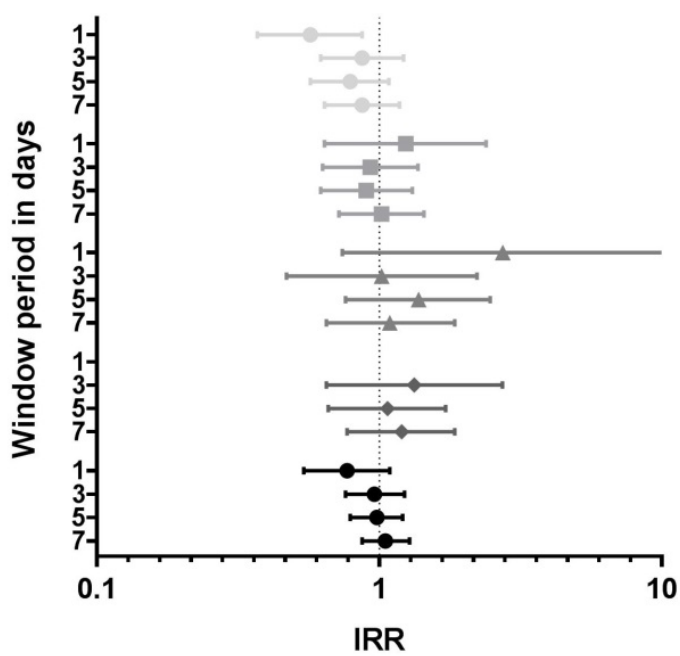

B) Hematological patients

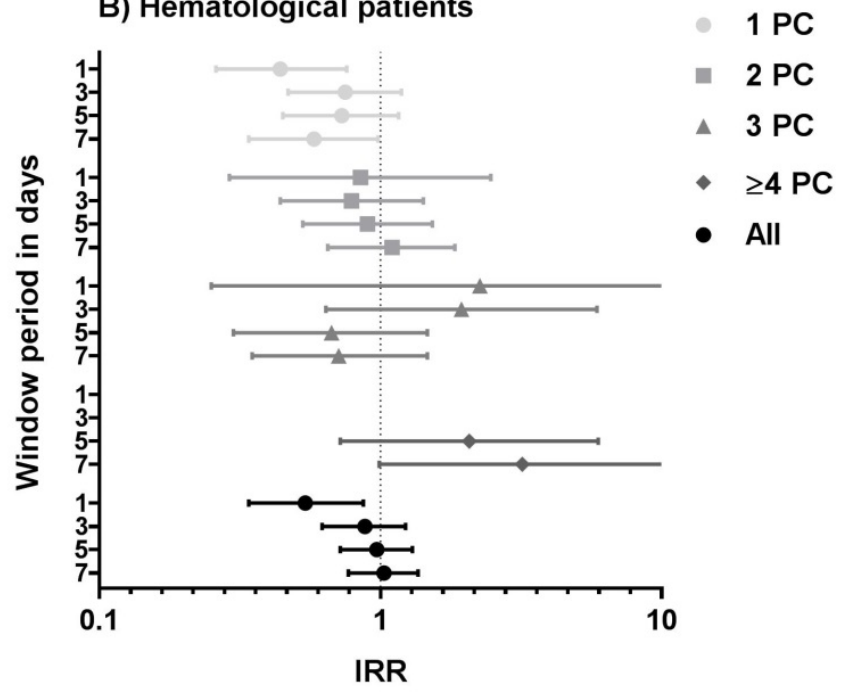

